\title{
Male brain ages faster: the age and gender dependence of subcortical volumes
}

\author{
András Király ${ }^{1} \cdot$ Nikoletta Szabó $^{1,2} \cdot$ Eszter Tóth $^{1} \cdot$ Gergő Csete $^{1} \cdot$ Péter Faragó $^{1}$. \\ Krisztián Kocsis $^{1}$ • Anita Must ${ }^{1} \cdot$ László Vécsei $^{1,3} \cdot$ Zsigmond Tamás Kincses $^{1,2}$
}

(C) Springer Science+Business Media New York 2015

\begin{abstract}
Effects of gender on grey matter (GM) volume differences in subcortical structures of the human brain have consistently been reported. Recent research evidence suggests that both gender and brain size influences volume distribution in subcortical areas independently. The goal of this study was to determine the effects of the interplay between brain size, gender and age contributing to volume differences of subcortical GM in the human brain. High-resolution T1-weighted images were acquired from 53 healthy males and 50 agematched healthy females. Total GM volume was determined using voxel-based morphometry. We used model-based subcortical segmentation analysis to measure the volume of subcortical nuclei. Main effects of gender, brain volume and aging on subcortical structures were examined using multivariate analysis of variance. No significant difference was found in total brain volume between the two genders after correcting for total intracranial volume. Our analysis revealed significantly larger hippocampus volume for females. Additionally, GM volumes of the caudate nucleus, putamen and thalamus displayed a significant age-related decrease in males as compared to females. In contrast to this only the thalamic volume
\end{abstract}

Electronic supplementary material The online version of this article (doi:10.1007/s11682-015-9468-3) contains supplementary material, which is available to authorized users.

Zsigmond Tamás Kincses

kincses.zsigmond.tamas@med.u-szeged.hu; http://www.nepsy. szote.u-szeged.hu/ kincsesz

1 Department of Neurology, Albert Szent-Györgyi Clinical Center, University of Szeged, Semmelweis u. 6, Szeged H-6725, Hungary

2 International Clinical Research Center, St. Anne's University Hospital Brno, Brno, Czech Republic

3 MTA-SZTE Neuroscience Research Group, Szeged, Hungary loss proved significant for females. Strikingly, GM volume decreases faster in males than in females emphasizing the interplay between aging and gender on subcortical structures. These findings might have important implications for the interpretation of the effects of unalterable factors (i.e. gender and age) in cross-sectional structural MRI studies. Furthermore, the volume distribution and changes of subcortical structures have been consistently related to several neuropsychiatric disorders (e.g. Parkinson's disease, attention deficit hyperactivity disorder, etc.). Understanding these changes might yield further insight in the course and prognosis of these disorders.

Keywords Subcortical structures $\cdot$ Brain volume $\cdot$ Gender . Aging $\cdot$ MRI

\section{Introduction}

Gender differences in behavioral aspects of addiction (Bisagno and Cadet 2014; Fattore et al. 2014), motor control (Kauranen and Vanharanta 1996; Ruff and Parker 1993), emotional memory (Cahill 2003) and several major neuropsychiatric disorders, including attention deficit hyperactivity disorder (ADHD) (Gershon 2002) and Parkinson's disease (Bourque et al. 2009) have been recently reported. Accordingly, the sexual dimorphism of the human brain anatomy has gained attention with neuroimaging methods being widely used to detect these differences (Cahill 2006; Cosgrove et al. 2007; DeLacoste-Utamsing and Holloway 1982; Goldstein et al. 2001). Whereas males convergingly exhibit larger cerebral volumes (Gur et al. 1991; Sowell et al. 2007) and head sizes (Scahill et al. 2003), females generally have a thicker cortex in several regions of the brain (Luders et al. 2006; Sowell et al. 2007). 
There is much less evidence on the sexual dimorphism of subcortical grey matter (GM) structures including the amygdala, caudate nucleus, accumbens, hippocampus, amygdala, pallidum, putamen and thalamus (Ahsan et al. 2007; Filipek et al. 1994). Considering that the basal ganglia nuclei possess a high density of sex steroid receptors (for reviews, see: (Taber et al. 2001), (Gray and Bingaman 1996)), the effect of gender on the volume of these structures might be crucial. Nevertheless, results are somewhat contradictory with several studies reporting larger volumes of the caudate nuclei (Luders et al. 2009), hippocampus (Murphy et al. 1996) and thalamus in females (Murphy et al. 1996; Takahashi et al. 2011), and some with opposing results (Sullivan et al. 2004; Rijpkema et al. 2012). The amygdala (Cheng et al. 2009), pallidum and the putamen (Rijpkema et al. 2012) have been consistently found to be larger in males.

Research evidence confirms aging to be associated with decrease in whole-brain volume (Courchesne et al. 2000; Gur et al. 1991; Scahill et al. 2003), grey matter volume (Courchesne et al. 2000; Ge et al. 2002a; Good et al. 2001; Guttmann et al. 1998; Pell et al. 2008; Raz et al. 1997; C. D. Smith et al. 2007; Taki et al. 2004), cortical thickness (Sowell et al. 2007), as well as temporal lobe volume (Scahill et al. 2003) and the hippocampal and thalamic volumes (Good et al. 2001). Studies on effects of aging on white matter (WM) volume decrease are inconsistent: some studies did not find a significant effect of aging on WM changes (Good et al. 2001; Taki et al. 2004), while others reported an increase in volume until middle adulthood, followed by a decline (Courchesne et al. 2000; Ge et al. 2002a) and yet others concluded that there was a steady decline with progressing aging (Guttmann et al. 1998; Lemaitre et al. 2005; Taki et al. 2011). Two studies using voxel-based techniques that reported no overall significant effect of aging on WM volume did reveal a decline with age in some areas (Good et al. 2001; Taki et al. 2004). The combined effects of age and gender on the human brain have been assessed suggesting a more profound decline in GM volume in males (Ge et al. 2002a; Raz et al. 1997; Taki et al. 2004). However, research evidence is inconsistent on one hand (Lemaitre et al. 2005) and sparse on the other, especially considering the subcortical GM structures. Most of the studies focusing on subcortical nuclei applied a voxel-based morphometric approach to identify gender differences. The deformable surface model based segmentation approach what we used in the current analysis offers advantages over intensity based procedures especially in regions with low tissue contrast. While age, gender and head size (intracranial volume) are the most commonly included "nuisance" variables when performing neuroimaging analysis, studies vary as to which of these variables are included and which method is used for correction (Perlaki et al. 2014). These factors might widely account for the great variability in the results.

In the present study an automatized, deformable mesh based segmentation toolkit (FSL-FIRST) was used to extract subcortical structures from the brain. Partial brain volumes were extracted with an intensity-based segmentation toolkit (FSL-SIENAX). Multivariate analysis of covariance and correlation analysis were run to evaluate the following: (1) gender effects on subcortical GM volumes with and without normalization for skull size; (2) interactions between aging and gender affecting volume changes.

\section{Methods and materials}

\section{Participants}

Fifty-three healthy males (mean age: $31.08 \pm 10.03$ years) and fifty age-matched healthy females (mean age: $33.00 \pm 11.34$ years) with no history of any neurological or psychiatric disorder were included in the study (Table 1).

The study was approved by the Ethics Committee of University of Szeged (authority number: 87/2009). All enrolled participants provided their written informed consent.

\section{Image acquisition}

Imaging was carried out with a 1.5 T GE Signa Excite MRI scanner. High-resolution T1-weighted images (3D IRFSPGR: TR/TE/TI: 10.3/4.2/450 ms, flip angle: $15^{\circ}$, ASSET: 2, FOV: $25^{*} 25 \mathrm{~cm}$, matrix: $256^{*} 256$, slice thickness: $1 \mathrm{~mm}$ ) were acquired.

\section{Image processing}

Tools from the FMRIB Software Library (FSL, version 5.0; Oxford Centre for Functional MRI of the Brain (FMRIB), UK; www.fmrib.ox.ac.uk/fsl) were used for data processing.

\section{Comparison of basal ganglia and partial brain volumes}

We used FIRST, a model-based segmentation/registration tool for volume comparison of the subcortical structures of males and females (Patenaude et al. 2011). This approach uses deformable surface meshes specific to subcortical structures, namely the amygdala, caudate nucleus, hippocampus, pallidum, putamen and thalamus. Given the observed intensities in a T1-weighted image, FIRST searches through linear combinations of shape modes of variation for the most probable shape instance based on learned models. We decided not 
Table 1 Demographic data on the participant subjects

\begin{tabular}{|c|c|c|c|c|c|c|}
\hline & \multicolumn{3}{|l|}{ Male } & \multicolumn{3}{|l|}{ Female } \\
\hline & mean & $\pm \mathrm{SD}$ & range & mean & $\pm \mathrm{SD}$ & range \\
\hline $\mathrm{N}$ & 53 & - & - & 50 & - & - \\
\hline Age (years) & 31.08 & 10.03 & $21-58$ & 33.00 & 11.34 & $21-57$ \\
\hline Handedness (left) & 6 & - & - & 7 & - & - \\
\hline \multicolumn{7}{|l|}{ Volumes } \\
\hline Total intracranial $\left(\mathrm{mm}^{3}\right)$ & $1,192,105.89$ & $85,334.42$ & $972,761.85-1,381,750.59$ & $1,064,220.04$ & $84,530.88$ & $887,409.06-1,296,592.68$ \\
\hline Total brain $\left(\mathrm{mm}^{3}\right)$ & $1,165,217.63$ & $83,151.41$ & $948,950.34-1,342,429.91$ & $1,041,990.31$ & $82,652.01$ & $861,476.12-1,270,109.32$ \\
\hline Gray matter $\left(\mathrm{mm}^{3}\right)$ & $642,377.23$ & $46,905.62$ & $527,133.52-758,544.08$ & $579,578.16$ & $42,978.76$ & $495,802.20-674,384.25$ \\
\hline White matter $\left(\mathrm{mm}^{3}\right)$ & $522,840.39$ & $43,260.20$ & $421,816.82-608,111.83$ & $462,412.14$ & $44,986.73$ & $360,447.47-621,760.47$ \\
\hline Cortex $\left(\mathrm{mm}^{3}\right)$ & $501,307.86$ & $37,441.32$ & $410,306.09-593,261.73$ & $451,801.73$ & $35,256.74$ & $381,160.13-520,349.99$ \\
\hline
\end{tabular}

to include the nucleus accumbens due to inappropriate segmentation.

Specific entities of brain volume (total brain volume, total GM, white matter, cortical GM and ventricular cerebrospinal fluid) were compared between the two groups with and without normalization for total intracranial volume. Total intracranial volume as well as grey and white matter volumes were estimated using SIENAX (S. M. Smith et al. 2001; S. M. Smith et al. 2002), an approach included in the FSL (S. M. Smith et al. 2004). SIENAX starts by extracting brain and skull images from the single whole-head input data (S. M. Smith 2002). The brain image is then affine-registered to MNI152 space (Jenkinson et al. 2002; Jenkinson and Smith 2001) using the skull image to determine the registration scaling, primarily to obtain the volumetric scaling factor (vscale), which is then used as normalization for head size. Subsequently, tissue-type segmentation with partial volume calculation is carried out (Zhang et al. 2001) in order to calculate the total volume of brain tissue, including separate estimates of grey and white matter partial volumes.

The raw and the normalised volumes of the subcortical structures were compared between the groups. For normalisation, the FIRST output volumes were multiplied by the vscale factor, obtaining normalised GM volumes of subcortical structures - as if skull size would have been identical for all participants.

Additionally, left/right volume ratios of subcortical structures were compared between groups. A ratio larger than 1 indicates larger structures on the left, while smaller than 1 larger structures on the right.

Multiple univariate analysis of variance with age as covariant (MANCOVA) was applied for statistical analysis (IBM SPSS Statistics 20). Correlations between volumes of subcortical structures, grey/white matter ratio, partial brain volumes and age were calculated for both groups (IBM SPSS Statistics 20). The results were Bonferroni-corrected ( $\mathrm{p}_{\text {corr }}$ ) and $p<0.05$ was chosen as the significance threshold.
For voxel-based morphometric analysis of the cortical gray matter, please see: Supplementary material.

\section{Results}

\section{Gender differences of partial brain volumes and subcortical structures - raw data}

MANCOVA ( mean age of comparison $=32.02$ years) revealed that all subcortical structures (max. $\left.\mathrm{P}_{\text {corr }}<0.002\right)$ and all partial brain volumes (max. $p<0.002$ ) without normalization for skull size were significantly larger in the male than in the female group (Fig. 1). The grey/white matter ratio did not reveal a significant difference $(p<0.077)$.

No significant group difference was found between left/right volume ratios of subcortical structures. However, it is noteworthy that volumes of the right caudate nucleus $\left(\mathrm{p}_{\text {corr }}<0.052\right)$ and the left thalamus $\mathrm{p}_{\text {corr }}<0.049$ ) were significantly larger than those of the corresponding contralateral structures in the male group only..

\section{Gender differences of partial brain volumes and subcortical structures - skull size normalised data}

After normalization for skull size, MANCOVA (mean age of comparison $=32.02$ years) revealed significantly larger subcortical GM volumes for the left $\left(\mathrm{p}_{\text {corr }}<0.011\right)$ and right hippocampus $\left(\mathrm{p}_{\text {corr }}<0.010\right)$ in the female group. Strikingly, the total $(p<0.003)$ and the cortical GM were also found to be relatively more extended in the female group as compared to males $(p<0.009)$ (Fig. 2).

In the male group, the volumes of the right caudate nucleus $\left(\mathrm{p}_{\text {corr }}<0.047\right)$ and the left thalamus $\left(\mathrm{p}_{\text {corr }}<0.015\right)$ were found to remain significantly larger than the contralateral pair of these structures. 


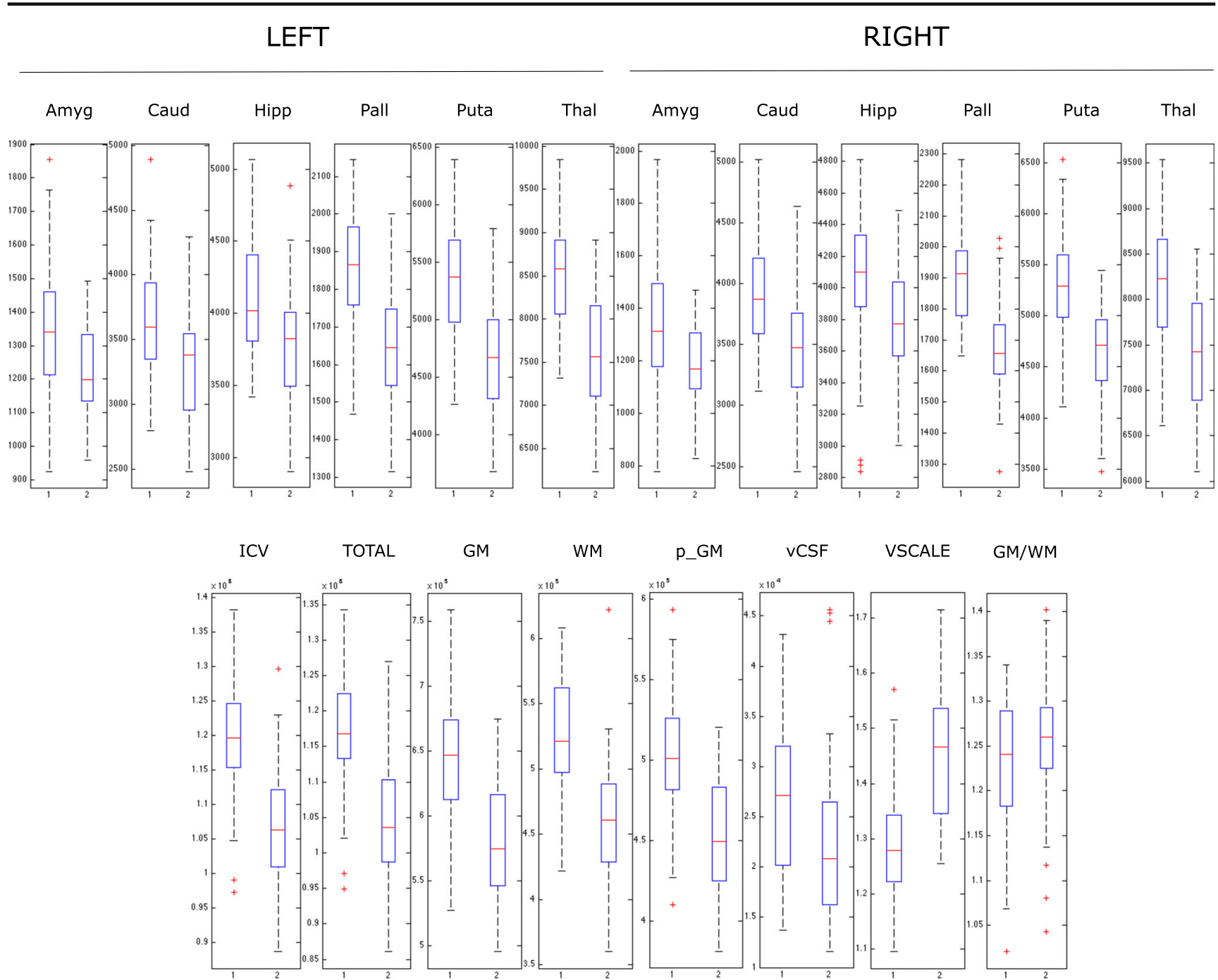

Fig. 1 Gender differences in the raw volumes

\section{Correlation of total, partial and subcortical GM volumes with age - raw data}

In the male group, total GM volumes $(\mathrm{R}=-0.366$, $p<0.0071)$, cortical GM volumes $(\mathrm{R}=-0.332, p<0.015)$ and the right thalamus $\left(\mathrm{R}=-0.365, \mathrm{p}_{\text {corr }}<0.043\right)$ showed a significant negative correlation with age when corrected for multiple comparisons. As for the volume of the left thalamus a tendency to a significant negative correlation with age $\left(\mathrm{R}=-0.345, \mathrm{p}_{\mathrm{corr}}<0.069\right)$ was detected.

In the female group, volumes of total GM $(\mathrm{R}=-0.425$, $p<0.002)$, cortical GM ( $\mathrm{R}=-0.418, p<0.003)$, the right hippocampus $\left(\mathrm{R}=-0.411, \mathrm{p}_{\text {corr }}<0.018\right)$ as well as the left $\left(\mathrm{R}=-0.373, \mathrm{p}_{\mathrm{corr}}<0.045\right)$ and the right thalamus $\left(\mathrm{R}=-0.439, \mathrm{p}_{\mathrm{corr}}<0.008\right)$ showed a significant negative correlation with age.

The grey/white matter ratio was found to correlate negatively with age for both males $(\mathrm{R}=-0.476, p<0.00032)$ and females $(\mathrm{R}=-0.397, p<0.004)$. However, the age-related grey/white matter ratio was found to be higher for females $(p<0.016)$.

Interestingly, the left/right volume ratio of the hippocampus exhibited a significant positive correlation with age in the female group only $\left(\mathrm{R}=0.509, \mathrm{p}_{\text {corr }}<0.00094\right)$.

\section{Correlation of total, partial and subcortical GM volumes with age - skull size normalised data}

In the male group, total brain volume $(\mathrm{R}=-0.507$, $p<0.00011)$, total $\left(\mathrm{R}=-0.685, p<10^{-6}\right)$ and cortical GM $\left(\mathrm{R}=-0.616, p<10^{-6}\right)$, left $\left(\mathrm{R}=-0.393, \mathrm{p}_{\mathrm{corr}}<0.021\right)$ and right caudate nucleus volume $\left(\mathrm{R}=-0.376, \mathrm{p}_{\mathrm{corr}}<0.033\right)$, left $\left(\mathrm{R}=-0.384, \mathrm{p}_{\mathrm{corr}}<0.0274\right)$ and right putamen $(\mathrm{R}=-0.408$, $\left.\mathrm{p}_{\text {corr }}<0.014\right)$ as well as left $\left(\mathrm{R}=-0.489, \mathrm{p}_{\text {corr }}<0.0012\right)$ and right thalamus volume $\left(\mathrm{R}=-0.508, \mathrm{p}_{\text {corr }}<0.0006\right)$ showed a significant negative correlation with age (Figs. 3 and 4).

In the female group a significant negative correlation with age was revealed for total brain volume $(\mathrm{R}=-0.373$, 


\section{LEFT}

RIGHT
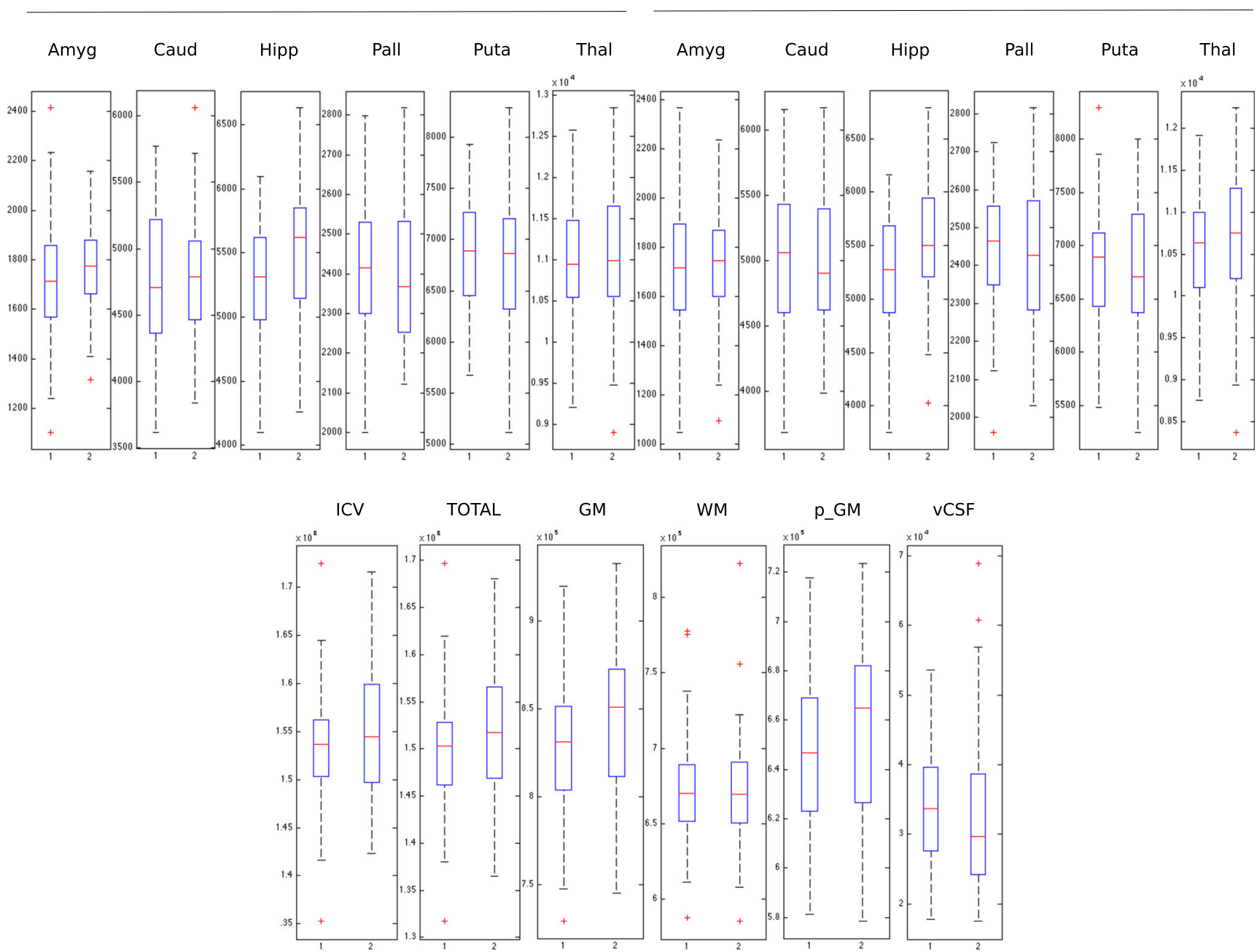

Fig. 2 Gender differences in the normalised volumes

$p<0.0076)$, total $(\mathrm{R}=-0.525, p<0.00009)$ and cortical GM $(\mathrm{R}=-0.516, p<0.00013)$, left $\left(\mathrm{R}=-0.399, \mathrm{p}_{\text {corr }}<0.024\right)$ and right thalamus $\left(\mathrm{R}=-0.452, \mathrm{p}_{\text {corr }}<0.006\right)$ (Figs. 3 and 4$)$.

Interestingly, the decline with age in normalized GM volume occurred at a faster pace in the group of males than for females $(\mathrm{z}=2.21, p<0.0271)$ (Fig. 3).

\section{Discussion}

In the current study we aimed to identify gender effects on subcortical GM volumes as well as interactions between aging and gender affecting volume changes in the human brain.

To our best knowledge, this study is the first to report the effect of interplay between gender and aging accounting for differences in head size on subcortical structures using a model-based segmentation tool. The main strength of our study consists in the large size of our cohort with homogeneous acquisition and analyses procedures.
In general, male brains were found to be larger than females', with larger grey and white matter as well as subcortical structures. However, most of these differences disappear when skull size is accounted for. As a result of correction for total intracranial volume we found females to have larger cortical and subcortical GM volume. Noteworthy, the volume of the hippocampus was found significantly larger in the female group as compared to males. We also detected a significant effect of hemisphere in the male group only, with larger volmes of the right caudate and the left thalamus as compared to their contralateral structures.

More importantly, we found an age dependent decrease in the the volume of cortical as well as subcortical grey matter. Latter remained significant after correction for skull size in the caudate, putamen and thalamus bilaterally for males and the thalamus bilaterally for females. Within the age-range of 21 to 58 years we found a linear decrease in GM volume with aging. Strikingly, this process proved to occur at a faster pace in males. 

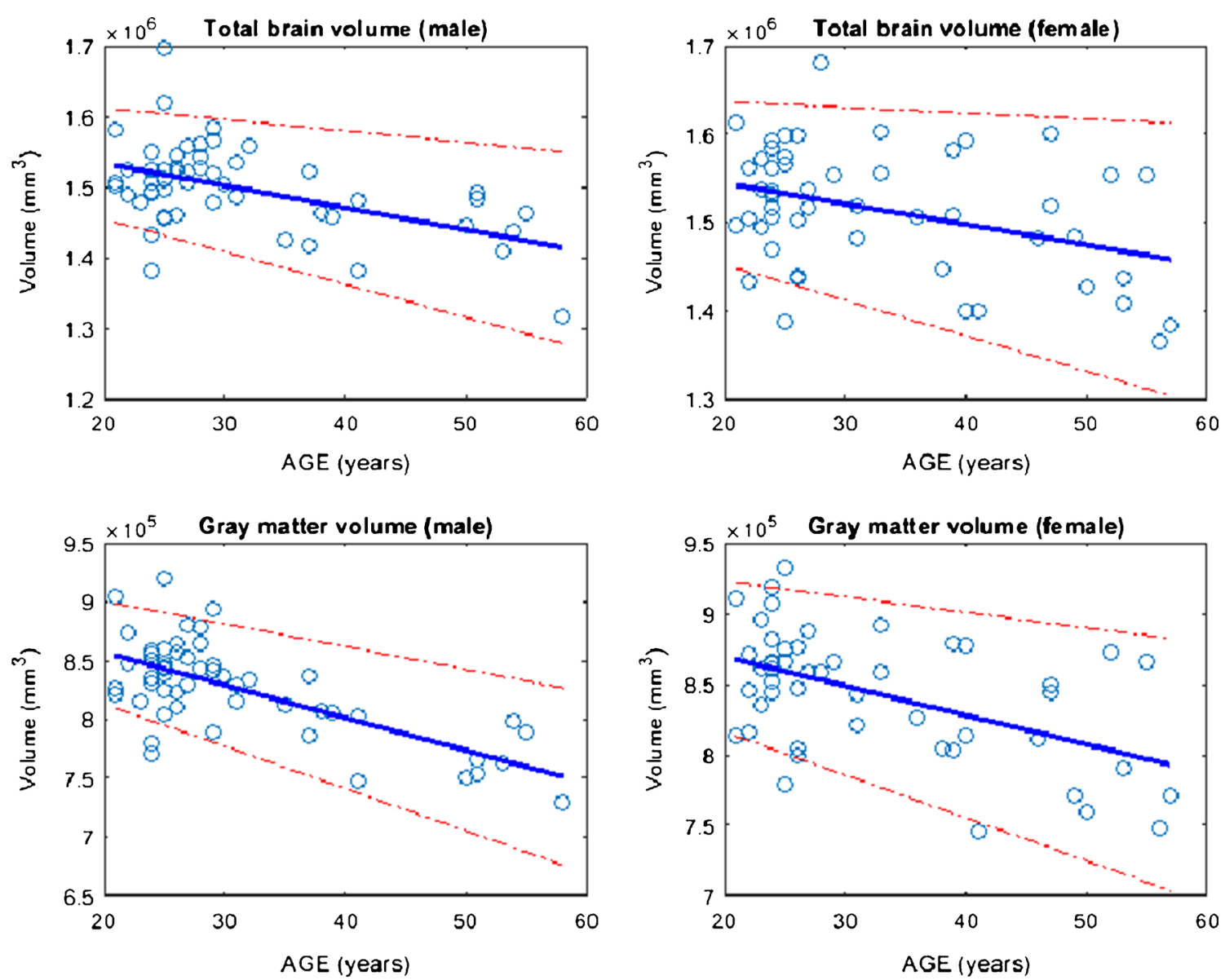

Fig. 3 Age-related decline in the normalized brain volumes in males and females

We propose the use and importance of our current findings to be two-fold: (1) methodological considerations of the investigation of subcortical structures and (2) potential functional implications related to gender and GM decline with age.

(1) While there is converging research evidence for total brain and GM volume to decline with age (Barnes et al. 2010; Courchesne et al. 2000; Ge et al. 2002b; C. D. Smith et al. 2007; Lemaitre et al. 2005; Takahashi et al. 2011), analysis approaches are typically different (Callaert et al. 2014). Volumes of the insular, medial frontal, posterior-parietal, occipital and temporal regions were shown to correlate negatively with age in an elderly population (C. D. Smith et al. 2007). Furthermore, a VBM study revelaed that cortical volume loss is not homogeneous (Takahashi et al. 2011).

Research evidence becomes much more sparse when focusing on the subcortical structures. Pevious VBM style analyses indicated a decline with age in the caudate and thalamic volumes (Callaert et al. 2014; C. D. Smith et al. 2007). However, the enlargement of the third and fourth ventricles might be a confounding variable in VBM analysis (C. D. Smith et al. 2007). Further studies applied manual tracing of subcortical structures identifying an age-related volume decline in the thalamus (Sullivan et al. 2004), the caudate and the putamen (Abedelahi et al. 2013). However, investigation was limited to certain structures and the relationship with age was observed for both genders. A recent study found significant brain size effects in the right amygdala and the bilateral caudate nucleus and significant gender effects in the bilateral putamen but no interactions between brain size and gender (Tang et al. 2013).

Our results echoed a previous study reporting an agerelated decline in the caudate, putamen and nucleus accumbens and a marginal effect in the thalamus in a similar agerange (35-60 years) (Goodro et al. 2012). However, the authors suggested that normalization to the cranium size accounted for the observed gender effect. Other studies have included gender as a nuisance variable in the general linear model analysis and found a negative correlation between age and the volumes of the hippocampus, amygdala, caudate and putamen (Barnes et al. 2010). Yet, other results were reported using Freesurfer, with a disproportionate degeneration of the subcortical volumes with aging ( $\mathrm{Li}$ et al. 2014). Our current results regarding the hippocampus are in agreement with previous reports of larger relative hippocampal size in females with the use of FIRST, also confirmed by a VBM style analysis (Kauranen and Vanharanta 1996).

We propose that the inconsistency of findings about gender and age-related differences in subcortical GM volumes might stem from methodological differences and relatively small 

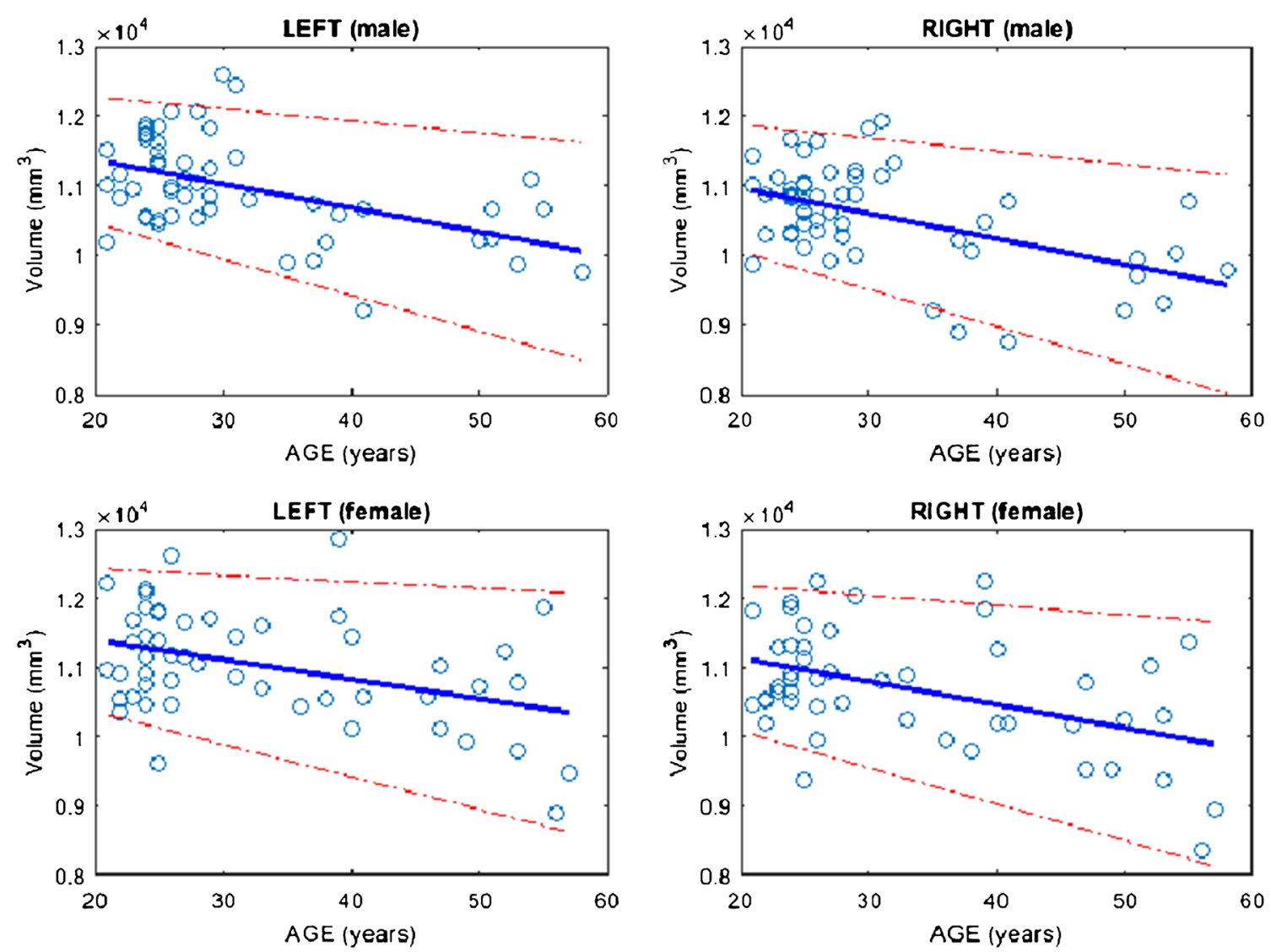

Fig. 4 Age-related decline in the normalized thalamic volumes in males and females

sample sizes. Most of the studies applied a VBM approach to identify gender differences in subcortical nuclei. While VBM is an excellent tool for the investigation of focal grey matter density differences, deformable surface model approaches such as FIRST are directly tuned for the volumetric analysis of the subcortical structures. Furthermore, in MRI studies, age, gender and head size (intracranial volume) are the most commonly included "nuisance" variables, though studies greatly vary as to which of the variables are included and which method is used for correction (Perlaki et al. 2014). Nevertheless, it is important to communicate transformation approach and group intracranial volume considerations when reporting structural findings of subcortical GM since it might carry several implications for interpretation of the results (Schwab et al. 2014).

In contrast with FIRST, the VBM analysis in our study did not detected any effects of age or gender on the subcortical structures (supplementary material). While the VBM approach is based on tissue-type/locally-averaged GM segmentation, FIRST utilises shape and intensity information jointly (Patenaude et al. 2011).

(2) The second aspect involves the underlying cellular, molecular and functional mechanisms of the age and gender related decline of GM volume. Primarily, neuronal and synaptic pruning has been proposed to play a critical role (Webb et al.
2001). However, findings of post-mortem histological studies suggested, that it is rather the size than the number of the individual cells explaining the age-related decline of GM (Terry et al. 1987; Peters et al. 1998). At the molecular level the expression of NoGo-A, a myelin-associated neurite growth inhibitor protein decreases with age (Kumari and Thakur 2014). Recent results also imply the impact of aging on GM/ WM diffusion changes, explaining some cognitive variability and even decline (Salminen et al. 2015).

The background of the disproportionate GM volume changes in males and females has not yet been elucidated, but the changes in hormone levels and the consequent sensitivity of the brain to hormonal effects are most certainly involved (Barron and Pike 2012). Above the structural differences, there is increasing evidence for the functional sexual dimorphism of subcortical structures. Amphetamine has been shown to cause a higher level of dopamine release in the male striatum, which correlates with the behavioural effect of the drug (Riccardi et al. 2011; Munro et al. 2006). Hippocampusrelated memory functions are differently affected by stress in males and females (Guenzel et al. 2014). Peripartum hormonal changes are known to modulate the hippocampal function (Galea et al. 2014). In addition to gender effects, recent evidence supports the influence of brain hemisphere showing lateralization of structure-function relationships, as well as 
more specific relationships between individual structures (e.g., left hippocampus) and functions relevant to particular aptitudes (e.g. vocabulary) (Jung et al. 2014). Our current results revealed a significant effect of hemisphere in the male group only, with larger volmes of the right caudate and the left thalamus as compared to their contralateral structures. It might be hypothesized that this difference relates to handedness, however, we did not find such a relationship. A recent study examining the deep GM of healthy adults by using magnetic susceptibility-weighted imaging did not reveal an association with handedness (Liu et al. 2013).

Considering that the volume of subcortical GM critically impacts the size of neurons, glia cells and number of synapses it entails, we might hypothesis that this also affects the function and performance of these structures. It is clear that deducing motor, cognitive and affective functional activity of subcortical GM solely from their structural characteristics would be inadmissibly simplified. Furthermore, observing changes in volume of subcortical GM influenced by gender and aging might yield better insight and with furhter investigations, even explain some clinically significant differences in males and females in several neurological and psychiatric conditions, e.g. Alzheimer's dementia (Qian et al. 2014), Parkinson's disorder (Gillies et al. 2014; Geevarghese et al. 2015), headache disorders (Macgregor et al. 2011), multiple sclerosis (Greer and McCombe 2011), major depression and bipolar disorder (MacMaster et al. 2014). Strikingly, some recent findings suggest that the volume of certain subcortical nuclei is even associated with crucial psychiatric conditions such as suicidal behavior (Gifuni et al. 2015). Further investigation of functional and behavioral correlates of accurately identified subcortical structures might have crucial implications for preventive measures and treatment of related disorders.

Acknowledgments The study was supported by the MTA-SZTE Neuroscience Research Group, the project FNUSA-ICRC (no. CZ.1.05/ 1.1.00/02.0123) from the European Regional Development Fund, by European Union - project ICRC-ERA-HumanBridge (No. 316345), the National Brain Research Program (Grant No. KTIA_13_NAP-A-II/20.) and an OTKA [PD 104715] grant.

\section{References}

Abedelahi, A., Hasanzadeh, H., Hadizadeh, H., \& Joghataie, M. T. (2013). Morphometric and volumetric study of caudate and putamen nuclei in normal individuals by MRI: Effect of normal aging, gender and hemispheric differences. Pol J Radiol, 78(3), 7-14. doi:10. 12659/PJR.889364.

Ahsan, R. L., Allom, R., Gousias, I. S., Habib, H., Turkheimer, F. E., Free, S., et al. (2007). Volumes, spatial extents and a probabilistic atlas of the human basal ganglia and thalamus. NeuroImage, 38(2), 261-270. doi:10.1016/j.neuroimage.2007.06.004.

Andersson, J. L. R., Jenkinson, M., \& Smith, S. (2007). Non-linear optimisation. FMRIB technical report. Oxford.
Ashburner, J., \& Friston, K. J. (2000). Voxel-based morphometry-the methods. NeuroImage, 11(6 Pt 1), 805-821. doi:10.1006/nimg. 2000.0582 .

Barnes, J., Ridgway, G. R., Bartlett, J., Henley, S. M., Lehmann, M., Hobbs, N., et al. (2010). Head size, age and gender adjustment in MRI studies: a necessary nuisance? NeuroImage, 53(4), 1244-1255. doi:10.1016/j.neuroimage.2010.06.025.

Barron, A. M., \& Pike, C. J. (2012). Sex hormones, aging, and alzheimer's disease. Frontiers in Bioscience (Elite Edition), 4, 976-997.

Bisagno, V., \& Cadet, J. L. (2014). Stress, sex, and addiction: potential roles of corticotropin-releasing factor, oxytocin, and arginine-vasopressin. Behavioural Pharmacology, 25(5-6), 445-457. doi:10. 1097/FBP.0000000000000049.

Bourque, M., Dluzen, D. E., \& Di Paolo, T. (2009). Neuroprotective actions of sex steroids in parkinson's disease. Frontiers in Neuroendocrinology, 30(2), 142-157. doi:10.1016/j.yfrne.2009.04. 014.

Cahill, L. (2003). Sex-related influences on the neurobiology of emotionally influenced memory. Annals of the New York Academy of Sciences, 985, 163-173.

Cahill, L. (2006). Why sex matters for neuroscience. Nature Reviews. Neuroscience, 7(6), 477-484. doi:10.1038/nrn1909.

Callaert, D. V., Ribbens, A., Maes, F., Swinnen, S. P., \& Wenderoth, N. (2014). Assessing age-related gray matter decline with voxel-based morphometry depends significantly on segmentation and normalization procedures. Frontiers in Aging Neuroscience, 6, 124. doi:10. 3389/fnagi.2014.00124.

Cheng, Y., Chou, K. H., Decety, J., Chen, I. Y., Hung, D., Tzeng, O. J., et al. (2009). Sex differences in the neuroanatomy of human mirrorneuron system: a voxel-based morphometric investigation. Neuroscience, 158(2), 713-720. doi:10.1016/j.neuroscience.2008. 10.026 .

Cosgrove, K. P., Mazure, C. M., \& Staley, J. K. (2007). Evolving knowledge of sex differences in brain structure, function, and chemistry. Biological Psychiatry, 62(8), 847-855. doi:10.1016/j.biopsych. 2007.03.001.

Courchesne, E., Chisum, H. J., Townsend, J., Cowles, A., Covington, J., Egaas, B., et al. (2000). Normal brain development and aging: quantitative analysis at in vivo MR imaging in healthy volunteers. Radiology, 216(3), 672-682. doi:10.1148/radiology.216.3. r00au37672.

DeLacoste-Utamsing, C., \& Holloway, R. L. (1982). Sexual dimorphism in the human corpus callosum. Science, 216(4553), 1431-1432.

Fattore, L., Melis, M., Fadda, P., \& Fratta, W. (2014). Sex differences in addictive disorders. Frontiers in Neuroendocrinology, 35(3), 272284. doi:10.1016/j.yfrne.2014.04.003.

Filipek, P. A., Richelme, C., Kennedy, D. N., \& Caviness Jr., V. S. (1994). The young adult human brain: an MRI-based morphometric analysis. Cerebral Cortex, 4(4), 344-360.

Galea, L. A., Leuner, B., \& Slattery, D. A. (2014). Hippocampal plasticity during the peripartum period: influence of sex steroids, stress and ageing. Journal of Neuroendocrinology. doi:10.1111/jne.12177.

Ge, Y., Grossman, R. I., Babb, J. S., Rabin, M. L., Mannon, L. J., \& Kolson, D. L. (2002a). Age-related total gray matter and white matter changes in normal adult brain. Part I: volumetric MR imaging analysis. AJNR. American Journal of Neuroradiology, 23(8), 13271333.

Ge, Y., Grossman, R. I., Babb, J. S., Rabin, M. L., Mannon, L. J., \& Kolson, D. L. (2002b). Age-related total gray matter and white matter changes in normal adult brain. Part II: quantitative magnetization transfer ratio histogram analysis. AJNR. American Journal of Neuroradiology, 23(8), 1334-1341.

Geevarghese, R., Lumsden, D. E., Hulse, N., Samuel, M., \& Ashkan, K. (2015). Subcortical structure volumes and correlation to clinical 
variables in parkinson's disease. Journal of Neuroimaging, 25(2), 275-280. doi:10.1111/jon.12095.

Gershon, J. (2002). A meta-analytic review of gender differences in ADHD. Journal of Attention Disorders, 5(3), 143-154.

Gifuni, A. J., Ding, Y., Olie, E., Lawrence, N., Cyprien, F., Le Bars, E., et al. (2015). Subcortical nuclei volumes in suicidal behavior: nucleus accumbens may modulate the lethality of acts. Brain Imaging and Behavior. doi:10.1007/s11682-015-9369-5.

Gillies, G. E., Pienaar, I. S., Vohra, S., \& Qamhawi, Z. (2014). Sex differences in parkinson's disease. Frontiers in Neuroendocrinology. doi:10.1016/j.yfrne.2014.02.002.

Goldstein, J. M., Seidman, L. J., Horton, N. J., Makris, N., Kennedy, D. N., Caviness Jr., V. S., et al. (2001). Normal sexual dimorphism of the adult human brain assessed by in vivo magnetic resonance imaging. Cerebral Cortex, 11(6), 490-497.

Good, C. D., Johnsrude, I. S., Ashburner, J., Henson, R. N., Friston, K. J., \& Frackowiak, R. S. (2001). A voxel-based morphometric study of ageing in 465 normal adult human brains. NeuroImage, 14(1 Pt 1), 21-36. doi:10.1006/nimg.2001.0786.

Goodro, M., Sameti, M., Patenaude, B., \& Fein, G. (2012). Age effect on subcortical structures in healthy adults. Psychiatry Research, 203(1), 38-45. doi:10.1016/j.pscychresns.2011.09.014.

Gray, T. S., \& Bingaman, E. W. (1996). The amygdala: corticotropinreleasing factor, steroids, and stress. Critical Reviews in Neurobiology, 10(2), 155-168.

Greer, J. M., \& McCombe, P. A. (2011). Role of gender in multiple sclerosis: clinical effects and potential molecular mechanisms. Journal of Neuroimmunology, 234(1-2), 7-18. doi:10.1016/j. jneuroim.2011.03.003.

Guenzel, F. M., Wolf, O. T., \& Schwabe, L. (2014). Sex differences in stress effects on response and spatial memory formation. Neurobiology of Learning and Memory, 109, 46-55. doi:10.1016/ j.nlm.2013.11.020.

Gur, R. C., Mozley, P. D., Resnick, S. M., Gottlieb, G. L., Kohn, M., Zimmerman, R., et al. (1991). Gender differences in age effect on brain atrophy measured by magnetic resonance imaging. Proceedings of the National Academy of Sciences of the United States of America, 88(7), 2845-2849.

Guttmann, C. R., Jolesz, F. A., Kikinis, R., Killiany, R. J., Moss, M. B., Sandor, T., et al. (1998). White matter changes with normal aging. Neurology, 50(4), 972-978.

Jenkinson, M., Bannister, P., Brady, M., \& Smith, S. (2002). Improved optimization for the robust and accurate linear registration and motion correction of brain images. NeuroImage, 17(2), 825-841.

Jenkinson, M., \& Smith, S. (2001). A global optimisation method for robust affine registration of brain images. Medical Image Analysis, 5(2), 143-156. doi: 10.1016/S1361-8415(01)00036-6

Jung, R. E., Ryman, S. G., Vakhtin, A. A., Carrasco, J., Wertz, C., \& Flores, R. A. (2014). Subcortical correlates of individual differences in aptitude. PloS One, 9(2), e89425. doi:10.1371/journal.pone. 0089425.

Kauranen, K., \& Vanharanta, H. (1996). Influences of aging, gender, and handedness on motor performance of upper and lower extremities. Perceptual and Motor Skills, 82(2), 515-525. doi:10.2466/pms. 1996.82.2.515

Kumari, A., \& Thakur, M. K. (2014). Age-dependent decline of nogo-a protein in the mouse cerebrum. Cellular and Molecular Neurobiology. doi:10.1007/s10571-014-0088-z.

Lemaitre, H., Crivello, F., Grassiot, B., Alperovitch, A., Tzourio, C., \& Mazoyer, B. (2005). Age- and sex-related effects on the neuroanatomy of healthy elderly. NeuroImage, 26(3), 900-911. doi:10.1016/j. neuroimage.2005.02.042.

Li, W., van Tol, M. J., Li, M., Miao, W., Jiao, Y., Heinze, H. J., et al. (2014). Regional specificity of sex effects on subcortical volumes across the lifespan in healthy aging. Human Brain Mapping, 35(1), 238-247. doi:10.1002/hbm.22168.
Liu, Y., Wang, G., Zhao, L., Geng, M., Wang, L., Bai, X., et al. (2013). SWI phase asymmetries in deep gray matter of healthy adults: is there an association with handedness? Brain Imaging and Behavior, 7(2), 220-226. doi:10.1007/s11682-012-9217-9.

Luders, E., Gaser, C., Narr, K. L., \& Toga, A. W. (2009). Why sex matters: brain size independent differences in gray matter distributions between men and women. The Journal of Neuroscience, 29(45), 14265-14270. doi:10.1523/JNEUROSCI.2261-09.2009.

Luders, E., Narr, K. L., Thompson, P. M., Rex, D. E., Woods, R. P., Deluca, H., et al. (2006). Gender effects on cortical thickness and the influence of scaling. Human Brain Mapping, 27(4), 314-324. doi:10.1002/hbm. 20187.

Macgregor, E. A., Rosenberg, J. D., \& Kurth, T. (2011). Sex-related differences in epidemiological and clinic-based headache studies. Headache, 51(6), 843-859. doi:10.1111/j.1526-4610.2011.01904.x.

MacMaster, F. P., Carrey, N., Langevin, L. M., Jaworska, N., \& Crawford, S. (2014). Disorder-specific volumetric brain difference in adolescent major depressive disorder and bipolar depression. Brain Imaging and Behavior, 8(1), 119-127. doi:10.1007/s11682-0139264-x.

Munro, C. A., McCaul, M. E., Wong, D. F., Oswald, L. M., Zhou, Y., Brasic, J., et al. (2006). Sex differences in striatal dopamine release in healthy adults. Biological Psychiatry, 59(10), 966-974. doi:10. 1016/j.biopsych.2006.01.008.

Murphy, D. G., DeCarli, C., McIntosh, A. R., Daly, E., Mentis, M. J., Pietrini, P., et al. (1996). Sex differences in human brain morphometry and metabolism: an in vivo quantitative magnetic resonance imaging and positron emission tomography study on the effect of aging. Archives of General Psychiatry, 53(7), 585-594.

Patenaude, B., Smith, S. M., Kennedy, D. N., \& Jenkinson, M. (2011). A bayesian model of shape and appearance for subcortical brain segmentation. NeuroImage, 56(3), 907-922. doi:10.1016/j. neuroimage.2011.02.046.

Pell, G. S., Briellmann, R. S., Chan, C. H., Pardoe, H., Abbott, D. F., \& Jackson, G. D. (2008). Selection of the control group for VBM analysis: influence of covariates, matching and sample size. NeuroImage, 41(4), 1324-1335. doi:10.1016/j.neuroimage.2008. 02.050 .

Perlaki, G., Orsi, G., Plozer, E., Altbacker, A., Darnai, G., Nagy, S. A., et al. (2014). Are there any gender differences in the hippocampus volume after head-size correction? A volumetric and voxel-based morphometric study. Neuroscience Letters, 570, 119-123. doi:10. 1016/j.neulet.2014.04.013.

Peters, A., Morrison, J. H., Rosene, D. L., \& Hyman, B. T. (1998). Feature article: are neurons lost from the primate cerebral cortex during normal aging? Cerebral Cortex, 8(4), 295-300.

Qian, S., Zhang, Z., Li, B., \& Sun, G. (2014). Functional-structural degeneration in dorsal and ventral attention systems for alzheimer's disease, amnestic mild cognitive impairment. Brain Imaging and Behavior. doi:10.1007/s11682-014-9336-6.

Raz, N., Gunning, F. M., Head, D., Dupuis, J. H., McQuain, J., Briggs, S. D., et al. (1997). Selective aging of the human cerebral cortex observed in vivo: differential vulnerability of the prefrontal gray matter. Cerebral Cortex, 7(3), 268-282.

Riccardi, P., Park, S., Anderson, S., Doop, M., Ansari, M. S., Schmidt, D., et al. (2011). Sex differences in the relationship of regional dopamine release to affect and cognitive function in striatal and extrastriatal regions using positron emission tomography and [(1)(8)F]fallypride. Synapse, 65(2), 99-102. doi:10.1002/syn. 20822 .

Rijpkema, M., Everaerd, D., van der Pol, C., Franke, B., Tendolkar, I., \& Fernandez, G. (2012). Normal sexual dimorphism in the human basal ganglia. Human Brain Mapping, 33(5), 1246-1252. doi:10. 1002/hbm.21283.

Ruff, R. M., \& Parker, S. B. (1993). Gender- and age-specific changes in motor speed and eye-hand coordination in adults: normative values 
for the finger tapping and grooved pegboard tests. Perceptual and Motor Skills, 76(3 Pt 2), 1219-1230. doi:10.2466/pms.1993.76.3c. 1219.

Salminen, L. E., Conturo, T. E., Laidlaw, D. H., Cabeen, R. P., Akbudak, E., Lane, E. M., et al. (2015). Regional age differences in gray matter diffusivity among healthy older adults. Brain Imaging and Behavior. doi:10.1007/s11682-015-9383-7.

Scahill, R. I., Frost, C., Jenkins, R., Whitwell, J. L., Rossor, M. N., \& Fox, N. C. (2003). A longitudinal study of brain volume changes in normal aging using serial registered magnetic resonance imaging. Archives of Neurology, 60(7), 989-994. doi:10.1001/archneur.60.7. 989.

Schwab, N. A., Tanner, J. J., Nguyen, P. T., Schmalfuss, I. M., Bowers, D., Okun, M., et al. (2014). Proof of principle: transformation approach alters caudate nucleus volume and structure-function associations. Brain Imaging and Behavior. doi:10.1007/s11682-0149332-X.

Smith, C. D., Chebrolu, H., Wekstein, D. R., Schmitt, F. A., \& Markesbery, W. R. (2007). Age and gender effects on human brain anatomy: a voxel-based morphometric study in healthy elderly. Neurobiology of Aging, 28(7), 1075-1087. doi:10.1016/j. neurobiolaging.2006.05.018.

Smith, S. M. (2002). Fast robust automated brain extraction. Human Brain Mapping, 17(3), 143-155. doi:10.1002/hbm.10062.

Smith, S. M., De Stefano, N., Jenkinson, M., \& Matthews, P. M. (2001). Normalized accurate measurement of longitudinal brain change. Journal of Computer Assisted Tomography, 25(3), 466-475.

Smith, S. M., Jenkinson, M., Woolrich, M. W., Beckmann, C. F., Behrens, T. E., Johansen-Berg, H., et al. (2004). Advances in functional and structural MR image analysis and implementation as FSL. NeuroImage, 23(Suppl 1), S208-S219. doi:10.1016/j.neuroimage. 2004.07.051.

Smith, S. M., \& Nichols, T. E. (2009). Threshold-free cluster enhancement: addressing problems of smoothing, threshold dependence and localisation in cluster inference. NeuroImage, 44(1), 83-98. doi:10. 1016/j.neuroimage.2008.03.061.

Smith, S. M., Zhang, Y., Jenkinson, M., Chen, J., Matthews, P. M., Federico, A., et al. (2002). Accurate, robust, and automated longitudinal and cross-sectional brain change analysis. NeuroImage, 17(1), 479-489. doi:10.1006/nimg.2002.1040.
Sowell, E. R., Peterson, B. S., Kan, E., Woods, R. P., Yoshii, J., Bansal, R., et al. (2007). Sex differences in cortical thickness mapped in 176 healthy individuals between 7 and 87 years of age. Cerebral Cortex, 17(7), 1550-1560. doi:10.1093/cercor/bhl066.

Sullivan, E. V., Rosenbloom, M., Serventi, K. L., \& Pfefferbaum, A. (2004). Effects of age and sex on volumes of the thalamus, pons, and cortex. Neurobiology of Aging, 25(2), 185-192.

Taber, K. H., Murphy, D. D., Blurton-Jones, M. M., \& Hurley, R. A. (2001). An update on estrogen: higher cognitive function, receptor mapping, neurotrophic effects. Journal of Neuropsychiatry and Clinical Neurosciences, 13(3), 313-317. doi:10.1176/Appi. Neuropsych.13.3.313.

Takahashi, R., Ishii, K., Kakigi, T., \& Yokoyama, K. (2011). Gender and age differences in normal adult human brain: voxel-based morphometric study. Human Brain Mapping, 32(7), 1050-1058. doi:10. 1002/hbm.21088.

Taki, Y., Goto, R., Evans, A., Zijdenbos, A., Neelin, P., Lerch, J., et al. (2004). Voxel-based morphometry of human brain with age and cerebrovascular risk factors. Neurobiology of Aging, 25(4), 455463. doi:10.1016/j.neurobiolaging.2003.09.002.

Taki, Y., Thyreau, B., Kinomura, S., Sato, K., Goto, R., Kawashima, R., et al. (2011). Correlations among brain gray matter volumes, age, gender, and hemisphere in healthy individuals. PloS One, 6(7), e22734. doi:10.1371/journal.pone.0022734.

Tang, T., Jiao, Y., Wang, X., \& Lu, Z. (2013). Gender versus brain size effects on subcortical gray matter volumes in the human brain. Neuroscience Letters, 556, 79-83. doi:10.1016/j.neulet.2013.09. 060 .

Terry, R. D., DeTeresa, R., \& Hansen, L. A. (1987). Neocortical cell counts in normal human adult aging. Annals of Neurology, 21(6), 530-539. doi:10.1002/ana.410210603.

Webb, S. J., Monk, C. S., \& Nelson, C. A. (2001). Mechanisms of postnatal neurobiological development: implications for human development. Developmental Neuropsychology, 19(2), 147-171. doi:10. 1207/S15326942DN1902 2.

Zhang, Y., Brady, M., \& Smith, S. (2001). Segmentation of brain MR images through a hidden markov random field model and the expectation-maximization algorithm. IEEE Transactions on Medical Imaging, 20(1), 45-57. doi:10.1109/42.906424. 